\title{
ALTERED INTERACTIONS OF BIOACTIVE FACTORS IN PRE-ECLAMPSIA
}

\author{
CHATTOPADHYAY A.B. ${ }^{1}$, SHARMA R.C. ${ }^{*}$, SHARMA R.K. ${ }^{2}$, SHARMA S. ${ }^{3}$ AND SHARMA R. ${ }^{4}$ \\ ${ }^{1}$ AIMST University, Semeling, 08100Bedong, Kedah, Malaysia. \\ 2Padmashree Dr D.Y. Patil Medical College, Quatre Bornes, Mauritius. \\ ${ }^{3}$ RVMS Hospital, Nalasopara, Mumbai- 401203, MS, India. \\ ${ }^{4} B M C$ Medical College, Sagar- 470 001, MP, India. \\ *Corresponding Author: Email- drsharmax@gmail.com
}

Received: April 02, 2013; Accepted: April 22, 2013

\begin{abstract}
Pre-eclampsia is a pregnancy specific syndrome characterised with hypertension, proteinuria and in severe cases manifest as hepatic, renal or CNS symptoms. Extensive studies on the etiological factors behind said syndrome have been carried out for the last three decades, however concrete evidence based information on its aetiopathogenesis is yet to be presented. The manifestations of Pre-Eclampsia, generally seen after 20 weeks of gestation, have been reported due to amalgamation of two major pathological events namely placental hypoperfusion and endothelial cell injury and leading to the advancement of the disease. The main objective of this study is to review the recent reports \& hypothesis regarding aetiology and pathophysiology of Pre-eclampsia and present an overview on viability of reported hypothesis based on the cumulative opinion to provide evidenced based knowledge on the various factors that may contribute and cause the aforesaid syndrome. The review suggests that development of preeclampsia involves interaction of many physiological processes resulting from altered hemodynamics attributed to circulatory biomarkers which in turn produce profound influence on blood flow and arterial blood pressure regulation. We also hypothesize that complete profile of vasoactive biomarkers needs to be evaluated in order to explore their pathophysiological role in aetiopathogenesis of preeclampsia. This paper thus provides scope for further research to study the effects of interplay of uteroplacental factors in the pathophysiology of preeclampsia.
\end{abstract}

Keywords- Pre-eclampsia, fetoplacental monitoring, uteroplacental factors, 6-ketoPGF 1alpha, plasma renin activity, aetiopathogenesis

Citation: Chattopadhyay A.B., et al. (2013) Altered Interactions of Bioactive Factors in Pre-Eclampsia. International Journal of Medical and Clinical Research, ISSN: 0976-5530 \& E-ISSN: 0976-5549, Volume 4, Issue 1, pp.-248-251.

Copyright: Copyright@2013 Chattopadhyay A.B., et al. This is an open-access article distributed under the terms of the Creative Commons Attribution License, which permits unrestricted use, distribution and reproduction in any medium, provided the original author and source are credited.

\section{Introduction}

Pre-eclampsia is a pregnancy specific syndrome manifesting usually after 20 weeks of gestation, as proteinuria, hypertension and other associated systemic symptoms. It is a perplexing disorder of pregnancy, occurs as end stage feto-placental unit response and is one of the leading causes of maternal morbidity and mortality in the world. It is estimated that approximately $5-7 \%$ of total pregnancies are associated with preeclampsia [1]. It was observed by Thadani, et al. [2] that pre eclampsia alone was responsible for approximately $15 \%$ premature deliveries and $17.6 \%$ maternal death in the United States, and for approximately $14 \%$ maternal deaths worldwide. Apart from the palliative and symptomatic therapies used in treatment of pre eclampsia, the only definite cure is delivery. In view of this fact, it is observed that pre eclampsia is associated with a high rate of maternal and neonatal mortality and morbidity. Though it still remains to be one of the common complications of pregnancy, little is certain about pathogenesis or prevention of preeclampsia. This disease of pregnancy is characterised by hypertension and proteinuria affecting both mothers and their fetus and cured only by delivery. The only tool regarding its diagnosis and management currently available with the obstetrician is "Effective monitoring of preeclampsia through prenatal care" and has helped in reducing maternal mortality, but maternal morbidity remains to be the same [3]. Furthermore, effects of this disorder influence considerably fetal mortality and morbidity and accounts for preterm delivery. The author of the this paper has reviewed the available resources in pubmed, biocentral, google researchgate and medscape on pathophysiology, aetiopathogenesis and role of biomarkers in preeclampsia and attempt to present a overview of interaction of many physiological processes resulting from altered hemodynamics attributed to circulatory biomarkers which in turn produce profound influence on blood flow and arterial blood pressure regulation.

\section{Preeclampsia, Pathophysiology}

Though the exact aetiology of pre eclampsia is not as yet defined, it is postulated with evidences that there are two defining factors leading to pre eclampsia, namely uteroplacental hypo-perfusion and diffuse endothelial cell injury [4]. These two events lead to hypertension, renal and hepatic symptoms. In severe cases the preeclampsia may progress to eclampsia which is essentially complicated with 
CNS symptoms, commonest being seizures.

The onset of pre eclampsia has been reported due to utero placental ischaemia which causes invasion of abnormal cytotrophoblast in the spiral arteries which in turn leads to the dysfunction of maternal vascular endothelium causes increased formation of vasoactive substances (endothelin and thromboxane) responsible for increased vascular sensitivity to angiotensin II and decrease in the circulating levels of vasodilators like nitric oxide and prostacyclin responsible for vasodilatation of circulatory bed. Thus it is the imbalance between vasoconstrictor and vasodilator substance associated with altered sensitivity of vasoactive substances [increased action of angiotensin II] might be the causes of impaired renal pressure and subsequent natriuresis as well as causing an increase in the total peripheral resistance may be the resultant cause of hypertension and proteinuria. The placenta also plays an important role by impaired production of proangiogenic and antiangiogenic factors. The proangiogenic factors include vascular endothelial growth factor (VEGF) and placental growth factor (PIGF), whereas the antiangiogenic factors include soluble VEGF receptor type I (sFlt-1) and soluble endoglin (sEng) etc. which have been found to contribute to produce clinical symptoms as seen in eclampsia. Further studies on other substances, measured in circulatory fluid, reports that beside the placental factors being in main focus for their role in pre eclampsia, other substances such as interleukins, oxidants, syncytial knots [5] and tumor necrosis factor [6] have also been postulated to play a role in development of pre eclampsia.

This paper focuses mainly on the recent studies demonstrating the relationship between placental factors and endothelial function responsible for development of preeclampsia to provide an overview of possible mechanisms which may ultimately causes altered hemodynamics leading to abnormal blood flow and arterial blood pressure regulation.

\section{Placental Factors}

Since preeclampsia is said to be a disease due to uteroplacental ischemia, extensive research were undertaken to identify and define the role of proangiogenic and anti-angiogenic factors released from placenta. It suggested that imbalance between pro and antiangiogenic factors might be the cause of preeclampsia. Karumanchi, et al. [7] found that there were elevated levels of sFlt-1 in both the plasma and amniotic fluid in preeclamptic patients and hypothesised that sFlt-1 levels may be useful as a predictive trait in diagnosis of pre eclampsia as it is seen to be elevated prior to onset of symptoms. There is extensive clinical evidence to suggest that there is a link between the imbalance between proangiogenic factors (VEGF \& PIGF) and antiangiogenic factors (sFlt-1) and the incidence of pre eclampsia. It was also reported that exogenous administration of sFlt-1 via adenovirus mediated gene transfer in pregnant experimental animals such as mice, rats and baboons $[8,9]$ demonstrated elevated arterial pressure and proteinuria like preeclamptic patients associated with a decreased plasma levels of VEGF and PIGF concentrations. In recent studies It was observed that an infusion of VEGF in pregnant rats with over expression of sFIt-1, leads to attenuation of the elevated BP and renal damage [10]. These observations thus suggest that there is definite relationship between an imbalance in angiogenic factors and the development of sign and symptoms of preeclampsia. However none of the above studies have been able to explain the mechanism by which sFlt-1 over expression occurs. Levine et al reported another antiangiogenic factor namely soluble endoglin (sEng), responsible for impaired transforming growth factor beta (TGF-B) binding to cell surface receptors, which in turn causes an inhibition of in vitro endothelial cell formation. It was reported that increase in activity of sFlt-1 and sEng together may be the leading cause of fetal growth restriction, proteinuria and severe hypertension $[11,12]$.

\section{Antioxidents}

Nitric Oxide (NO), produced by the endothelial cells of the blood vessels is known potent vascular relaxant also reported to have important role in development of preeclampsia $[13,14]$, because its increased production by the placental endothelial cells causes lowering of blood pressure attributed to relaxation of vascular smooth muscle cells. Endothelial cell injury as it is observed in preeclampsia, impairs the ability of blood vessels to relax, thus may have role in development of preeclampsia. Significantly elevated level of NO in normal pregnancy is said to be responsible for the cardiovascular adaptations of pregnancy. A study reported that there is increase in the asymmetrical dimethylarginine (ADMA), which is a NO synthetase inhibitor before the onset of clinical symptoms of pre eclampsia. These observation suggest that NO can be used as an indicator to assess the degree of uteroplacental ischemia before it precipitate the development of preeclampsia. Alexandar et al has reported that pregnant rats with placental ischaemia did not demonstrate increase in urinary nitrite or nitrate levels as compared to the control subjects [15]. It was also reported in a study by Conrad et al that there was no evidence for a decrease in NO production in a carefully monitored environment with control over the dietary intake. Lower NO release from isolated vascular strips of pregnant rats with placental ischaemia was also seen in another study [16]. Oxidative stress attributed to placental factors leading to an imbalance of pro and anti-oxidant factors, causing endothelial dysfunction directly or through mediators, has been reported to play a role in genesis of preeclampsia [17]. In pre-eclamptic patients, increased expression of Dihydronicotinamide adenine dinucleotide phosphate (NADPH) oxidase was observed as compared to the pregnant women. Moreover a higher level of placental NADPH oxidase has been seen in women with early onset of preeclampsia as compared with those with late onset [18], suggesting that early onset preeclampsia is more influenced by placental factors than late onset disease. There were reports that in preeclampsia, the levels of vitamin C, E A, beta carotene, glutathione and iron binding protein are lower as compared to normal pregnant but supplement therapy of Vitamin $C$ and $\mathrm{E}$ during pregnancy failed to reduce risk of preeclampsia, IUGR and still birth [19]. Hence it is derived from these observations that lower level of these nutritional supplements may have their vascular effects attributed to antioxidant deficiency but it will be premature to suggest that use of these supplements as prophylactic strategic line of treatment may reduce the risk of preeclampsia.

\section{Prostacyclin}

Prostacyclin is a prostanoid synthesized from the endoperoxide in the fetal and maternal musculature. Unlike other prostaglandin, $P G I 2$ is not inactivated during its passage through pulmonary circulation, hence it is known to exert its effects on local as well on systemic vasculature. It has been reported that prostacyclin has a strong vasodilator effect, partially mediated by attenuation of vasoconstriction caused by Angiotensin II [20]. Prostacyclin, being a potent vasodilator and having anti-platelet aggregating properties has been reported to alter hemodynamic changes in fetal and maternal circulation. In pregnancy-induced hypertension, prostacyclin production reported to be reduced or unchanged but its production 
has been critically examined. Since pre-eclampsia is characterized by increased sensitivity to pressor effect of Angiotensin II, associated with increased platelet aggregation and reduced Uteroplacental blood flow, the authors of the present papers measured the plasma level of 6-keto prostaglandin $\mathrm{F}$ as indicator of Prostacyclin activity $\left(\mathrm{PGI}_{2}\right.$ ) and plasma Renin Activity (PRA) in normal pregnant women and pre-eclamptic women, with a aim to find out co-relation if any between the $\mathrm{PGI}_{2}$ \& pre-eclampsia. It was observed that PGI2 activity (mean 6-keto PGF1a level) as well as Plasma renin Activity (PRA) in cases of pre-eclampsia were found to be significantly lower than that of pregnant women $(P<.001)$ and non pregnant group $(P<001)$. Prostacyclin level $(6-k e t o$ PGF1a) and PRA level in pre eclampsia also demonstrated gradual decrease in $\mathrm{PGI}_{2}$ activity with severity of the diseases suggesting an inverse relation between $\mathrm{PGI}_{2}$ and severity [21]. We therefore suggest that reduced production of prostacyclin has been attributed partly to limited availability of enzyme responsible for conversion of $\mathrm{PGH}_{2}$ in to $\mathrm{PGI}_{2}$. It has been shown that $\mathrm{PGH} 2$ conversion in to $\mathrm{PGI} 2$ requires prostacyclin synthetase [22]. Reduced oxygen availability to placental tissue resulting from uteroplacental ischaemia could also be linked in mechanism of defective prostacyclin production [23]. Reduced arachidonic acid in fetal circulation may account for decreased production of prostacyclin in feto-maternal unit. Reduced PRA in pre eclampsia indicates suppression of renin release mechanism in the kidney, which has been attributed to, decreased prostacyclin production or sodium \& water retention, a feature of preeclampsia, may suppress the release of renin from kidney and cause fall in PRA. Decreased concentration of renin substrate could also be another factor for suppression of PRA in preeclampsia. These observation demonstrate that deficient prostacyclin in pre eclampsia may be a key player in pathogenesis of pre eclampsia and provide a pathophysiologic explanation for the clinical features of the disease. However further studies needs to be carried to identify the genetic defect in vascular endothelium to determine altered pattern of enzymatic or metabolic pathways causing release of vasodilator substances such as nitric oxide, prostacyclin and hyperpolarizing factor as well as vasoconstrictor factors such as endothelin, angiotensin II and thromboxane A2 from endothelium [24].

\section{Estrogens and Estrogen Metabolites}

Estrogens and estrogen metabolites have important functions in cardiovascular and reproductive physiology, yet the patterns of estrogen synthesis, metabolism, and the individual plasma profile of estrogens and estrogen metabolites during human pregnancy as well as in preeclampsia remain undetermined. We performed plasma measurement of $17 \beta$ estradiol in preeclampsia and human placental lactogen (HPL) and found statistically lowest level as compared to normal pregnant. We also correlated level of these hormones with severity of disease and observed inverse correlation between estradiol \& HPL with severity of preeclampsia [25]. Liquid chromatography mass spectrometry measurement of estradiol-17 $\beta$ in plasma samples from normotensive pregnant women and preeclampsia subjects were also have been reported to be significantly lower in women with preeclampsia. These findings demonstrate that aberrant estrogen synthesis and altered pattern of metabolism in preeclampsia might be due to changes in normal vascular function [26]. Assessment of ESRa expression using reverse transcription polymerase chain reaction (RT-PCR) analysis and western blot analysis in placenta of preeclampsia reveals that estradiol and estrogen receptors are deregulated, thus suggest the close link between estradiol and aetiopathogenesis of preeclampsia [27]. However further study of the altered physiological process of estrogen synthesis and its interaction with altered vascular physiology in pregnancy as observed in preeclampsia patients is highly desirable.

\section{Recent Bioactive Markers}

Effects of selected reproductive toxicants as isolated in environment has been found to have estrogenic activity and reported to produce profound effect on trophoblast cell line. It was found that cell line viability after exposure with these toxicants for $24 \mathrm{~h}$ was found to be decreased as compared to control [28]. Carolina et al in their in-vitro studies on effect of exposure of DDT, bisphenol A (EDCs) on proliferation of endometrial endothelial cells also reported that theses EDCs affect the viability of cell line and increase the proportion of necrotic cells leading to endometrial angiogenesis [29]. It is therefore necessary to explore the role of these reproductive toxicants in aetiopathogensis of preeclampsia. It is suggested that these environmental pollutants interfere with normal endocrine signaling pathways and disrupts the normal physiological neuroendocrine control system which may be responsible for sudden onset of preeclampsia. These findings raise concern about the negative, potential effects of various environmental polluting chemicals commonly referred as endocrine disrupting chemicals, on pregnancy success and fetal health.

In the recent years, visfatin, an enzymes secreted by the adipocytes, have been in the focus as biomarkers of preeclampsia. Visfatin has been reported to be involved in biosynthesis of nicotinamide adenine dinucleotide (NAD) and found to be decreased in preeclampsia [30]. However its diagnostic or prognostic potential are yet to be established as Frasshauer et al reported that level of visfatin in preeclampsia were just opposite to the report of $\mathrm{Hu}$, el at. [31] Adrenomedullin, known to cause prolonged hypotension mediated by vasodilation has been studied recently. It is said to be released from vascular and endothelial cells, mediate its action by regulating the vascular permeability [32]. It is reported that circulating level of adrenomedullin were elevated in number of disease conditions involving pathologic changes in vasculature [33]. A study by Senna et al demonstrated increased level of plasma adrenomedullin in normal preganancy [34]. However still more insight of the adrenomedullin needs to be explored prior to conclude its role as potential diagnostic or therapeutic agent.

\section{Summary}

In summary, development of sign and symptoms of preeclampsia appears to be the outcome of altered interaction of bioactive factors including genetic, dietary and environmental leading to placental ischemia consequently results in disrupting the endocrine signaling by the release of biologically active factors such as growth factors, cytokines, ROS, HIF-1 and antibodies to vasoconstrictor receptors. It is assumed that disturbance in the critical balance between production of the vasodilator mediators and release of the vasoconstrictive substances during pregnancy is the leading cause of development of preeclampsia. It is desirable to focus on understanding the cellular mechanism of release of these bioactive substances as well as causes of disruption of cell signaling which lead to critical imbalance between vasodilator and vasoconstrictors.

\section{References}

[1] Redman C.W., Sargent I.L. (2005) Science, 308, 1592-1594.

[2] Thadhani R.I., Johnson R.J., Karumanchi S.A. (2005) Hyperten- 
sion., 46, 1250-1251.

[3] Villar J., Carroli G., Wojdyla D., Abalos E., Giordano D., Ba'aqeel H., Farnot U., Bergsjø P., Bakketeig L., Lumbiganon P., Campodónico L., Al-Mazrou Y., Lindheimer M., Kramer M. (2006) Am. J. Obstet. Gynecol., 194(4), 921-931.

[4] Roberts J.M., Gammill H.S. (2005) Hypertension, 46(6), 12431249.

[5] Roberts J.M., Von Versen-Hoeynck F. (2007) Hypertension, 49, 15-16.

[6] LaMarca B.B., Bennett W.A., Alexander B.T., Cockrell K., Granger J.P. (2006) Hypertension, 46, 1022-1025.

[7] Karumanchi S.A., Bdolah Y. (2004) Endocrinology, 145, 48354837.

[8] Makris A., Thornton C., Thompson J., Thomson S., Martin R., Ogle R., Waugh R., McKenzie P., Kirwan P., Hennessy A. (2007) Kidney Int., 71, 977-984.

[9] Maynard S.E., Min J.Y., Merchan J., Lim K.H., Li J., Mondal S., Libermann T.A., Morgan J.P., Sellke F.W., Stillman I.E., Epstein F.H., Sukhatme V.P., Karumanchi S.A. (2003) J. Clin. Invest. 111, 649-658.

[10]Li Z., Zhang Y., Ying Ma J., Kapoun A.M., Shao Q., Kerr I., Lam A., O'Young G., Sannajust F., Stathis P., Schreiner G., Karumanchi S.A., Protter A.A., Pollitt N.S. (2007) Hypertension, 50, 686-692.

[11]Levine R.J., Lam C., Qian C., Yu K.F., Maynard S.E., Sachs B.P., Sibai B.M., Epstein F.H., Romero R., Thadhani R., Karumanchi S.A. (2006) N. Engl. J. Med., 355, 992-1005.

[12]Crews J.K., Herrington J.N., Granger J.P., Khalil R.A. (2000) Hypertension, 35, 367-372.

[13]Noris M., Todeschini M., Cassis P., Pasta F., Cappellini A., Bonazzola S., Macconi D., Maucci R., Porrati F., Benigni A., Picciolo C., Remuzzi G. (2004) Hypertension, 43, 614-622.

[14]Conrad K.P., Kerchner L.J., Mosher M.D. (1999) Am. J. Physiol., 277, F48-F57.

[15]Alexander B.T., Llinas M.T., Kruckeberg W.C., Granger J.P. (2004) Hypertension, 43, 832-836.

[16]Gandley R.E., Tyurin V.A., Huang W., Arroyo A., Daftary A., Harger G., Jiang J., Pitt B., Taylor R.N., Hubel C.A., Kagan V.E. (2005) Hypertension, 45, 21-27.

[17]Noris M., Todeschini M., Cassis P., Pasta F., Cappellini A., Bonazzola S., Macconi D., Maucci R., Porrati F., Benigni A., Picciolo C., Remuzzi G. (2004) Hypertension, 43, 614-622.

[18]Raijmakers M.T.M., Dechend R., Poston L. (2004) Hypertension, 44, 374-380.

[19]Rumbold A.R., Crowther C.A., Haslam R.R., Dekker G.A., Robinson J.S. (2006) New England Journal of Medicine, 354, 17961806.

[20]Goodman R.P., Killam A.R., Brash A.R. (1982) Am. J. Obste. Gynec., 142, 817.

[21]Sharma R.C. and Sharma R.K. (2012) Int. J. of Mole. Biol., 3(1), 40-42

[22]Gorden R.D., Persons S., Symonch E.M. (1996) Lancet, 1, 347.

[23]Walsh W.S. (1995) Am. J. Obste. Gynec., 152, 335.

[24]Tanbe A.F. and Khalil R.A. (2010) Curr. Bioact. Compd., 6(1),
60-75.

[25]Sharma R.C., Hazra D.K. and Sharma S. (1986) Journal of Med. Phy., 11(1), 5-10.

[26]Sheikh O.J., Chanel T.T., Magness R.R. (2013) Hypertension, 61, 480-487.

[27]Kumar P., Luo Y., Tudela C., Alexander J.M. and Mendelson C.R. (2013) Mol. Cell. Biol., 33(8).

[28]Bechi N., Sorda G., Spagnoletti A., Bhattacharjee J., Vieira Ferro E.A., de Freitas Barbosa B., Frosini M., Valoti M., Sgaragli G., Paulesu L., letta F. (2013) Toxicology in Vitro., 27 (3), 995-1186.

[29]Carolina Bredhult, Britt-Marie Bäcklin and Matts Olovsson (2007) Reproductive Toxicology, 23(4), 550-559.

[30]Hu W., Wang Z., Wang H., Huang H., Dong M. (2008) Acta. Obstet. Gynecol. Scand., 87, 413-418.

[31]Fasshauer M., Waldeyer T., Seeger J., Schrey S., Ebert T., Kratzsch J., Lossner U., Bluher M., Stumvoll M., Faber R., Stepan H. (2007) Clin. Endocrinol., 69(1), 69-73.

[32]Hinson J.P., Kapas S., Smith D.M. (2000) Endocr. Rev., 21, 138 $-167$.

[33]Kato J., Tsuruda T., Kita T., Kitamura K., Eto T. (2005) Arterioscler Thromb Vasc. Biol., 25, 2480-2487.

[34]Senna A.A., Zedan M., bd El Salam G.E., El Mashad A.I. (2008) Medscape J. Med., 10, 29. 